\title{
RKKY interaction in single-walled nanotubes
}

\author{
Vijay B. Shenoy* \\ Materials Research Centre, Indian Institute of Science, Bangalore 560 012, India \\ and Centre for Condensed Matter Theory, Indian Institute of Science, Bangalore 560 012, India
}

\begin{abstract}
The Ruderman-Kittel-Kasuya-Yoshida interaction between magnetic impurities in single-walled nanotubes is studied in the framework of a free electron model. It is found that the interaction decays inversely as axial separation between the impurities as in the case of a purely one-dimensional system. The key feature found is that the inverse-distance interaction is modulated by a nonperiodic function that depends on the size (radius) of the tube. Implications for magnetically doped nanotubes are discussed.
\end{abstract}

\section{INTRODUCTION}

Carbon nanotubes (CNTs), ever since their discovery by Ijima, ${ }^{1}$ have held the interest of scientists and technologists due to their amazing and unconventional properties. The exotic physical properties of CNTs include an elastic modulus exceeding that of steel by an order of magnitude, and electronic properties such as ballistic conduction brought about by a quasi one-dimensional geometry. ${ }^{2}$

CNTs can be single-walled or multi-walled. ${ }^{2}$ The structure of CNTs can be visualized as "roll up" of graphene sheets. This model not only explains the physical structure of CNTs but also provides for an understanding of the electronic structure. The nanotube is described by a chiral vector $C$ $=n \boldsymbol{a}_{1}+m \boldsymbol{a}_{2}$ whose magnitude is proportional to the tube diameter $\left(\boldsymbol{a}_{1}, \boldsymbol{a}_{2}\right.$ are the basis vectors of the graphene sheet $\left.{ }^{2}\right)$. The tube is metallic if $(n-m) / 3$ is an integer. ${ }^{2-5}$ These predictions have been verified experimentally, for example, by tunneling spectroscopy (see the review by Odom et al. ${ }^{6}$ and references therein).

Transport experiments on CNTs have resulted in a variety of observations. Metallic single-walled tubes (in the form of single tubes or ropes) are known to exhibit ballistic conduction, ${ }^{7}$ Coulomb blockade, ${ }^{8}$ Luttinger liquid behavior, ${ }^{9}$ etc., under different conditions. Semiconducting nanotubes exhibit high carrier mobilities. ${ }^{10}$ The ability to make both metallic and semiconducting nanotubes with the same technology, along with their attractive electronic properties, have provided on impetus to the emerging area of nanotube electronics. $^{11}$

The physics of magnetic impurities in a carbon nanotube was investigated experimentally by Odom et al. ${ }^{12}$ The presence of magnetic Co clusters on the nanotube produced a Kondo type resonance. These experiments have motivated several theoretical investigations of a single magnetic impurity in CNTs. ${ }^{13,14}$ In this context, an interesting question arises regarding the interaction of two spatially separated magnetic impurities in a metallic CNT, mediated by the conduction electrons. Understanding the physics of the magnetic interaction between impurities in a metallic CNT might provide clues to making magnetic carbon nanotubes which could find an application in spintronic devices. Further, it is of interest to study the dependence of the Ruderman-Kittel-
Kasuya-Yoshida (RKKY) interaction on the tube radius. The aim of this paper, therefore, is to address the question of the interaction between magnetic impurities in nanotubes.

The interaction between magnetic impurities, called the RKKY interaction, in solids is a well researched topic. It was first studied in the context of the exchange interaction between nuclear magnetic moments by Ruderman and Kittel ${ }^{15}$ followed by Kasuya ${ }^{16}$ and Yoshida ${ }^{17}$ in the context of the exchange interaction between magnetic impurities in solids. An insightful review by Van Vleck ${ }^{18}$ clarified many subtle issues relating to the RKKY interaction in three dimensions. The summary article by Kittel ${ }^{19}$ is especially useful in the present context since it discusses the RKKY interaction in different dimensions. This review ${ }^{19}$ contained an error (corrected in a very brief erratum) for the one-dimensional case and was later clarified by Yafet. ${ }^{20}$ All these calculations are based on the free electron model, and the main result is that at large separation $r$ between the impurities the interaction behaves as $T\left(2 k_{f} r\right) / r^{d}$ in $d$ dimensions where $k_{f}$ is the magnitude of the Fermi wave vector. The periodic function $T(\cdot)$ is $\cos (\cdot)$ in $d=1,3$ and $\sin (\cdot)$ in $d=2$. This work derives the RKKY interaction in nanotubes, especially the asymptotic behavior at large separation between the impurities. To this end, the electronic states in the nanotube are modeled using free electron theory since the long range behavior is unaffected by the details of the true band structure in metallic tubes.

The paper is organized as follows. The next section (Sec. II) contains the band structure of the free electron nanotube, and the calculation of the RKKY interaction in nanotubes. Results are presented in Sec. III; this section also contains the asymptotic result for the interaction at large separation. The paper is concluded with a discussion in Sec. IV. Details of calculations, not important to follow the main text, are relegated to the Appendix.

\section{CALCULATION OF RKKY INTERACTION IN NANOTUBES}

This section has two parts. The first part (Sec. II A) contains a discussion of the band structure of the free electron nanotube and is followed in the second (Sec. II B) by the calculation of the RKKY interaction. 


\section{A. Free electron nanotube: Electronic structure}

The single-walled nanotube considered here is of radius $R$ and axial periodicity $L$. Points on the tube are denoted by $(X, \theta)$, where $X$ is the axial coordinate and $\theta$ is the angle along the circumference of the tube. The single particle energy eigenstates are described by two quantum numbers $K$ and $n$ which represent the axial momentum (along the $X$ direction) and the rotational angular momentum. In terms of dimensionless variables $x=X / R, k=R K$, the energy eigenvalues and eigenstates are

$$
E_{K, n}=E_{o} \epsilon_{k, n}=\frac{\hbar^{2}}{2 m_{e} R^{2}}\left(k^{2}+n^{2}\right),
$$

( $m_{e}$ is the electron mass, $E_{o}=\hbar^{2} / 2 m_{e} R^{2}$ ) and

$$
\psi_{k, n}(x, \theta)=\frac{1}{\sqrt{2 \pi L}} e^{i(k x+n \theta)},
$$

where $k=2 \pi l R / L$, and $l$ and $n$ are integers.

The dimensionless density of states $g(\epsilon)$ is given by

$$
g(\epsilon)=\frac{1}{2 \pi}\left(\frac{L}{R}\right) \sum_{n=-\infty}^{\infty} \frac{\Theta\left(\epsilon-n^{2}\right)}{\sqrt{\epsilon-n^{2}}},
$$

where $\Theta(\xi)=1, \forall \xi>0,=0, \forall \xi \leqslant 0$. For a density $\Sigma$ (the number per unit area of the tube surface) of electrons, the dimensionless Fermi energy $\epsilon_{f}$ (chemical potential at zero temperature) is given by the solution of the equation

$$
\frac{1}{\pi} \sum_{n=-\infty}^{\infty} \Theta\left(\epsilon_{f}-n^{2}\right) \sqrt{\epsilon_{f}-n^{2}}=2 \pi \rho, \quad \rho=R^{2} \Sigma .
$$

Clearly, the physics of the free electron nanotube is governed by a single dimensionless number $\rho$ which is a product of the square of the nanotube radius $R$ and the electron density $\Sigma$. Since $\Sigma$ is usually fixed in nanotubes, $\rho$ is taken here to depend on the radius and is called the size parameter.

Figure 1 shows the dependence of the Fermi energy $\epsilon_{f}$ on the size parameter $\rho$. The kinks in the curve occur at those values of the size parameter at which new angular momentum bands become occupied. Associated with each occupied band, there is a Fermi wavevector given by

$$
k_{f n}=\sqrt{\epsilon_{f}-n^{2}}, \quad n=-N_{f}, \ldots, N_{f},
$$

and is shown schematically in the inset of Fig. 1. The highest occupied angular momentum band is denoted by $N_{f}$.

For a given $\rho$, the Fermi energy $\epsilon_{f}$, the highest occupied angular momentum band $N_{f}$, and the Fermi wave vectors $k_{f n}$ describe the electronic structure of the free electron nanotube.

\section{B. The RKKY interaction}

The RKKY interaction is obtained in two steps. ${ }^{21}$ First, the spin susceptibility of the nanotube free electron gas is determined using perturbation theory. The spin susceptibility is then used to determine the RKKY interaction.

To determine the spin susceptibility, the perturbing Hamiltonian is taken as

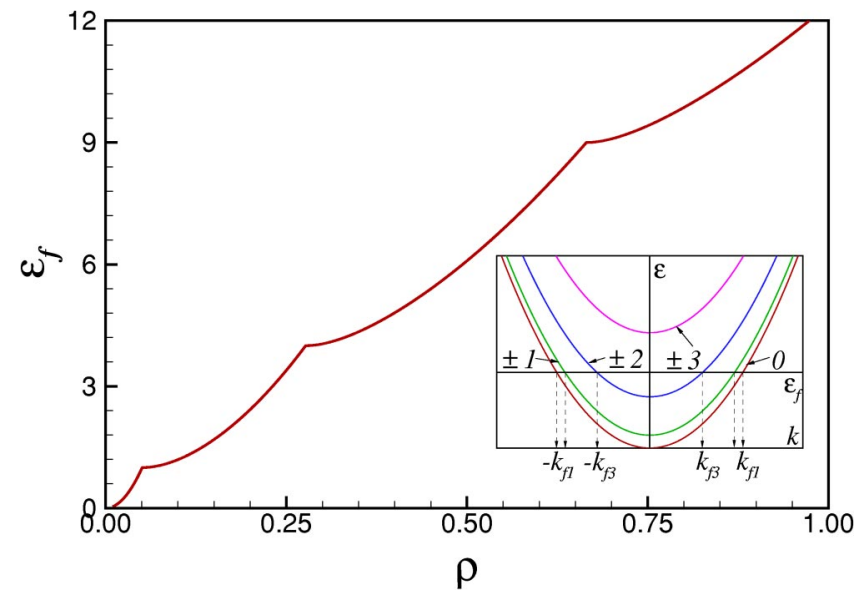

FIG. 1. (Color online) Dimensionless Fermi energy $\boldsymbol{\epsilon}_{f}$ as a function of the size parameter $\rho$. The inset shows schematically the Fermi wave vectors in different bands $\left(k_{f 2},-k_{f 2}\right.$ not shown). Angular momentum quantum numbers $(n)$ of different bands are as indicated in the inset.

$$
\mathcal{H}_{B}=-g \mu_{B} \boldsymbol{s} \cdot \boldsymbol{B}
$$

where $\boldsymbol{B}$ is the magnetic field, $\boldsymbol{s}$ is the electron spin operator and other symbols have usual meanings. ${ }^{21}$ The magnetic field may be inhomogeneous; in particular, for the calculation of the spin susceptibility, the magnetic field (only nonvanishing $z$ component is considered) is taken to have the form

$$
B_{z}(x, \theta)=\frac{1}{2}\left(B_{q, m} e^{-i(q x+m \theta)}+B_{-q,-m} e^{i(q x+m \theta)}\right) .
$$

The spin susceptibility due to the magnetic field (7) is calculated to be

$$
\begin{aligned}
\chi^{s}(q, m) & \\
= & \frac{g \mu_{B}}{8 \pi^{2} E_{0}} \sum_{n=-N_{f}}^{N_{f}} \int_{-k_{f n}}^{k_{f n}} d k\left(\frac{1}{\epsilon_{k-q, n-m}-\epsilon_{k, n}}+\frac{1}{\epsilon_{k+q, n+m}-\epsilon_{k, n}}\right) \\
= & \frac{g \mu_{B}}{8 \pi^{2} E_{0}} \sum_{n=-N_{f}}^{N_{f}} \frac{1}{2 q}\left(\ln \left|\frac{\left(q+k_{f n}\right)^{2}+(n-m)^{2}-\epsilon_{f}}{\left(q-k_{f n}\right)^{2}+(n-m)^{2}-\epsilon_{f}}\right|\right. \\
& \left.+\ln \left|\frac{\left(q+k_{f n}\right)^{2}+(n+m)^{2}-\epsilon_{f}}{\left(q-k_{f n}\right)^{2}+(n+m)^{2}-\epsilon_{f}}\right|\right)
\end{aligned}
$$

such that the spin density is $s(x, \theta)=\chi^{s}(q, m) B_{z}(x, \theta)$ for the magnetic field in (7).

The susceptibility pertaining to the RKKY interaction is obtained when the magnetic field is set to a delta function, i.e., $B_{z}(x, \theta)=B_{0} \delta(x) \delta(\theta)$. In this scenario, the spin density is $s(x, \theta)=\chi^{s}(x, \theta) B_{0}$. The susceptibility $\chi^{s}(x, \theta)$ is related to $\chi^{s}(q, m)$ via

$$
\chi^{s}(x, \theta)=\frac{1}{(2 \pi)^{2}} \sum_{m=-\infty}^{\infty} \int_{-\infty}^{\infty} d q e^{i(q x+m \theta)} \chi^{s}(q, m)
$$

The interaction of magnetic impurities with the conduction electrons is given by the $s-d$ type Hamiltonian ${ }^{22}$ as 


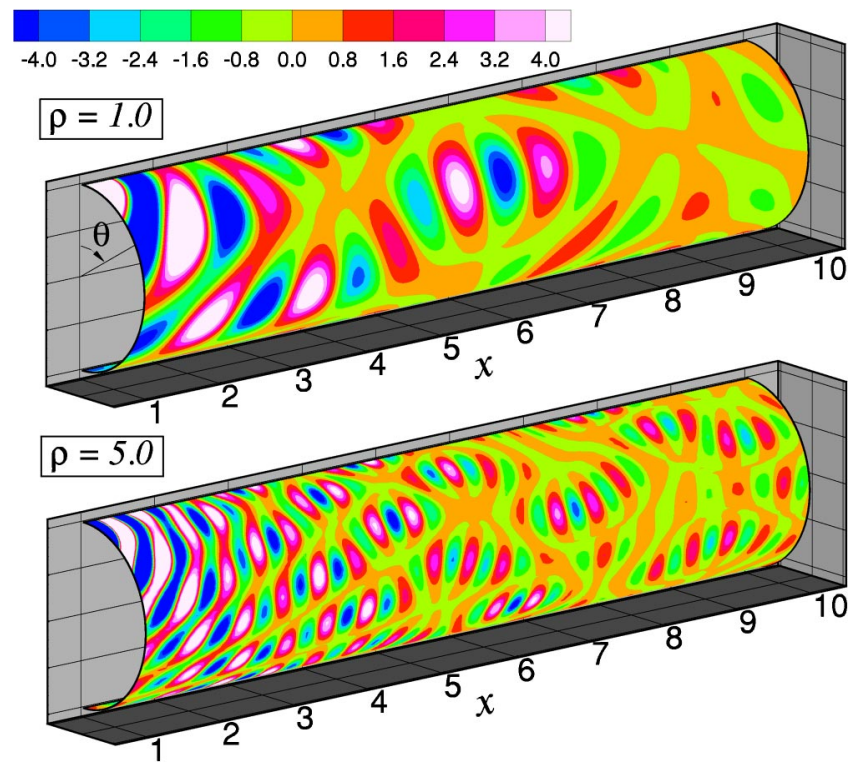

FIG. 2. (Color online) The dimensionless RKKY interaction $\mathcal{J}(x, \theta)$ for two different values of $\rho$. All lengths are normalized by the radius $R ; x=X / R$ is the dimensionless axial coordinate.

$$
\mathcal{H}_{s d}=-J \int d x d \theta s(x, \theta) \cdot \boldsymbol{S}(x, \theta),
$$

where $s(x, \theta)$ is the spin field and $S(x, \theta)$ is the impurity spin field given by

$$
S(x, \theta)=\sum_{p} S_{p} \delta\left(x-x_{p}\right) \delta\left(\theta-\theta_{p}\right)
$$

$\left(x_{p}, \theta_{p}\right)$ are the coordinates of the $p$ th impurity, and $S_{p}$ is its spin operator. $J$ is the magnetic coupling between the electron spin and the impurity spin. The second order perturbation theory of (10) gives the RKKY Hamiltonian for the impurity spins,

$$
\mathcal{H}_{\mathrm{RK} \mathrm{KY}}=\sum_{p, r} j\left(x_{p}-x_{r}, \theta_{p}-\theta_{r}\right) \boldsymbol{S}_{p} \cdot \boldsymbol{S}_{r} .
$$

The RKKY interaction $j(x, \theta)$ is related to the spin susceptibility (9) via

$$
j(x, \theta)=\frac{J^{2}}{g \mu_{B}} \chi^{s}(x, \theta)=\frac{J^{2}}{32 \pi^{4} E_{0}} \mathcal{J}(x, \theta) .
$$

The final result for the dimensionless RKKY interaction obtained using (9) is

$$
\mathcal{J}(x, \theta)=H_{0}(x)+2 \sum_{m=1}^{\infty} \cos (m \theta) H_{m}(x),
$$

where $H_{0}(x)$ and $H_{m}(x)$ are functions defined in (A12) (the Appendix).

\section{RESULTS}

Figure 2 shows contour plots of $\mathcal{J}(x, \theta)$ on the surface of the nanotube. Several points are noted. First, $\mathcal{J}(x, \theta)$ shows strong oscillations (positive sign implies anti-ferromagnetic coupling and negative, ferromagnetic). Second, the absolute value of the interaction falls with increasing $x$. Third, there is strong angular dependence even at large distances; $\mathcal{J}(x, \theta)$ is a strong function of $\theta$. Finally, with increasing $\rho$ (or increasing $R$ ), the short range behavior is expected to be like that of a two-dimensional system. This is indeed seen in the case of $\rho=5$ (Fig. 2) for $x$ near 1 and $\theta$ near 0 .

The interesting question at this juncture is the behavior of $\mathcal{J}(x, \theta)$ for large separation, $x \rightarrow \infty$. It is evident from (14) that the asymptotic behavior is determined by that of the functions $H_{m}(x)$. For all $m>M,(\mathrm{~A} 12)$ and (A11) show that $H_{m}(x)$ go to zero exponentially as $x \rightarrow \infty$. $M$ is calculated to be $N_{f}+\sqrt{\epsilon_{f}}$. With this observation and other results of the Appendix, the asymptotic behavior of $\mathrm{J}(x, \theta)$ is found to be

$$
\mathcal{J}^{a}(x, \theta)=\frac{T(x, \theta)}{x} .
$$

$T(x, \theta)$ is given by

$$
\begin{aligned}
& \frac{1}{2 \pi} T(x, \theta)=\sum_{n=-N_{f}}^{N_{f}} \frac{\cos \left(k_{f n} x\right)}{k_{f n}}+\sum_{m=1}^{M} \cos (m \theta)\left[\sum_{n=-N_{f}}^{N_{f}} \Theta\left(-\alpha_{+}\right)\right. \\
& \times\left(\frac{\cos \left(\left(k_{f n}-\sqrt{\left|\alpha_{+}\right|}\right) x\right)}{k_{f n}-\sqrt{\left|\alpha_{+}\right|}}+\frac{\cos \left(\left(k_{f n}+\sqrt{\left|\alpha_{+}\right|}\right) x\right)}{k_{f n}+\sqrt{\left|\alpha_{+}\right|}}\right) \\
& +\sum_{n=-N_{f}}^{N_{f}} \Theta\left(-\alpha_{-}\right)\left(\frac{\cos \left(\left(k_{f n}-\sqrt{\left|\alpha_{-}\right|}\right) x\right)}{k_{f n}-\sqrt{\left|\alpha_{-}\right|}}\right. \\
& \left.\left.+\frac{\cos \left(\left(k_{f n}+\sqrt{\left|\alpha_{-}\right|}\right) x\right)}{k_{f n}+\sqrt{\left|\alpha_{-}\right|}}\right)\right] \text {, }
\end{aligned}
$$

with $\alpha_{ \pm}=(n \pm m)^{2}-\epsilon_{f}$. It is noted that $T(x, \theta)$ is completely determined by the specification of $\rho$ which fixes $\epsilon_{f}$ etc. Further, for a given $\theta, T(x, \theta)$ is not a periodic function of $x$ since, in general, $k_{f n}$ and $k_{f n}-\sqrt{\left|\alpha_{ \pm}\right|}$are not multiples of a single wave vector $k_{0}$. (This is also true of $k_{f n} s$ for different values of $n$.) Thus, relation (15) shows that the RKKY interaction has an asymptotic nature similar to the 1D case with the crucial difference that the oscillating modulation function is not periodic and is determined by the size of the nanotube (via the parameter $\rho$ ). A comparison of (14) and (15) is shown in Fig. 3 for $\rho=1.0$.

Relations (14)-(16) are readily interpreted in terms of particle-hole excitations. The first term in (14) and (16) are terms whose contributions arise from particle-hole excitations that occur in the same angular momentum band, i.e., the intraband particle-hole excitations. The second term in (14) and (16) are contributions that arise from interband particle-hole excitations, and as is evident from (14), contributions to this arise from all possible angular momentum bands. The nonperiodicity of $T(x, \theta)$ may now be readily understood. As noted before, the Fermi wavevectors of the occupied bands $k_{f n}$ are not simple multiples of a common wavevector $k_{0}$. Thus, the intraband contribution to the interaction by itself leads to an asymptotic nonperiodic modulating function. The interband contribution further accentuates the nonperiodic behavior for the same reason $k_{f n}$ and $k_{f n}$ 


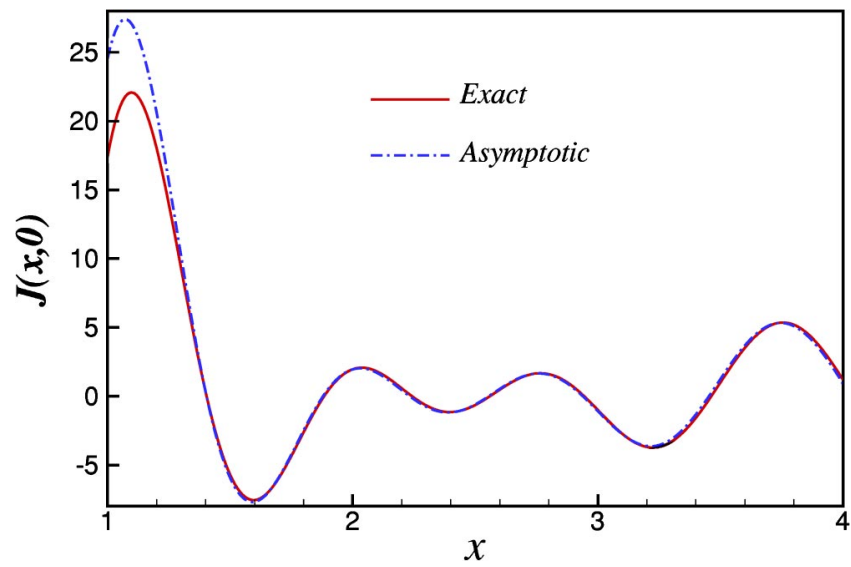

FIG. 3. (Color online) A comparison of $\mathcal{J}(x, 0)$ and $\mathcal{J}^{a}(x, 0)$ for $\rho=1.0$.

$-\sqrt{\left|\alpha_{ \pm}\right|}$are not multiples of a common $k_{0}$ ). It is also noted that the angular dependence of the interaction arises solely from the interband particle-hole excitations; this is another feature of the nanotube that is absent in the simple 1D case.

\section{DISCUSSION}

The main result of the present calculation is that the asymptotic behavior of the RKKY interaction in nanotubes scales as $T(x, \theta) / x$. All size effects (effects of radius $R$ ) are absorbed in the function $T(x, \theta)$. This is not altogether unexpected due to the quasi-one-dimensional nature of nanotubes. However, the new feature is that $T(x, \theta)$ is not a periodic function of $x$ for a given $\theta$.

The present calculation assumes that the magnetic impurities bind to the wall of the nanotube [as is evident from (11)]. However, the results can be easily generalized to other configurations. For example, if the impurities are located along the axis of the tube, the RKKY interaction is given by just the first term of (14); the asymptotic behavior is given by $T(x) / x$ where $T(x)$ is the (in general) nonperiodic function obtained from the first term of (16). Clearly, $T(x)$ is periodic only if $N_{f}=0$, i.e., only when the lowest angular momentum band is occupied; in the general case, $T(x)$ is nonperiodic. This may be contrasted with the purely one-dimensional result. $^{20}$

These results may have interesting implications for a long nanotube doped randomly with magnetic impurities. Since the function $T(x, \theta)$ is nonperiodic, it is possible that the effective coupling of a given impurity with all the other impurities effectively cancels. Thus, it is possible each magnetic impurity will behave independently of others, suggesting that a magnetically doped nanotube will not show strong magnetoresistance effects. At larger concentrations, the nonperiodic nature of $T(x, \theta)$ is likely to leave the magnetic impurities in a glassy state when the couplings do not cancel, suggesting that magnetically doped nanotubes could be interesting systems to study quasi-one-dimensional spin glasses.

The present work is based on a simple free electron picture which predicts the asymptotic behavior at large distances, and in particular obtains analytical results for the
RKKY interaction as a function of the tube radius. These results are not specific to carbon nanotubes, but applicable to any metallic single-walled tubes. In the specific case of CNT, as noted in the Introduction, the electronic structure is strongly influenced by the chirality of the tube, and not determined solely by the radius. Further detailed work is necessary to uncover the influence of chirality, and associated electronic band structure on the nature of the interaction and this issue will be addressed in a subsequent publication. While these factors will determine the short range behavior and the approach to the asymptotic limit, it is clear that such a detailed calculation will give long distance asymptotic relations similar to those derived here. Another promising line of investigation would be to probe the effect of electron interactions and associated correlation physics (Luttinger liquid behavior) on the RKKY interaction.

\section{ACKNOWLEDGMENTS}

Support for this work by the Indian National Science Academy under the Young Scientist Program is gratefully acknowledged. The author is grateful to Professor H. R. Krishnamurthy for illuminating discussions. Thanks are also due to T. Gupta, B. Mukherjee, S. Pathak, S. Saha, P. Sanyal, and S. Sengupta for suggestions.

\section{APPENDIX: MATHEMATICAL DETAILS}

\section{Functions $S(a, x)$ and $R(k, b, x)$}

In this section, two functions $S$ and $R$, necessary for the calculation of the RKKY interaction, are defined:

$$
S(a, x)=2 \pi \operatorname{sgn}(a)\left(\frac{\pi}{2}-\operatorname{Si}(|a| x)\right),
$$

where $\operatorname{sgn}(a)$ is the sign of $a$ and zero if $a=0$ :

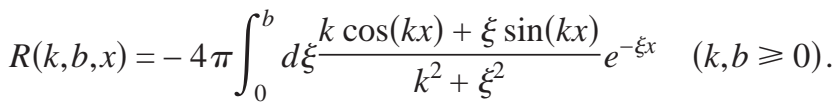

For $x \rightarrow \infty, S$ and $R$ have asymptotic formulas [up to $O\left(1 / x^{2}\right)$ ]

$$
\begin{gathered}
S^{a}(a, x)=2 \pi \operatorname{sgn}(a)\left(\frac{\cos (|a| x)}{|a| x}+\frac{\sin (|a| x)}{a^{2} x^{2}}\right), \\
R^{a}(k, b, x)=-4 \pi\left(\frac{\cos (k x)}{k x}+\frac{\sin (k x)}{k^{2} x^{2}}\right) .
\end{gathered}
$$

\section{The function $G(k, \alpha, x)$}

The determination of $\chi^{s}(x, \theta)$ from $\chi^{s}(q, m)$ in (9) requires integrals of the form

$$
G(k, \alpha, x)=\int_{-\infty}^{\infty} d q \frac{e^{i q x}}{q} \ln \left|\frac{(q+k)^{2}+\alpha}{(q+k)^{2}+\alpha}\right|,
$$

with $k, x>0, \alpha$ any real number. This integral is evaluated separately for the two cases, $\alpha \leqslant 0$ and $\alpha>0$. 


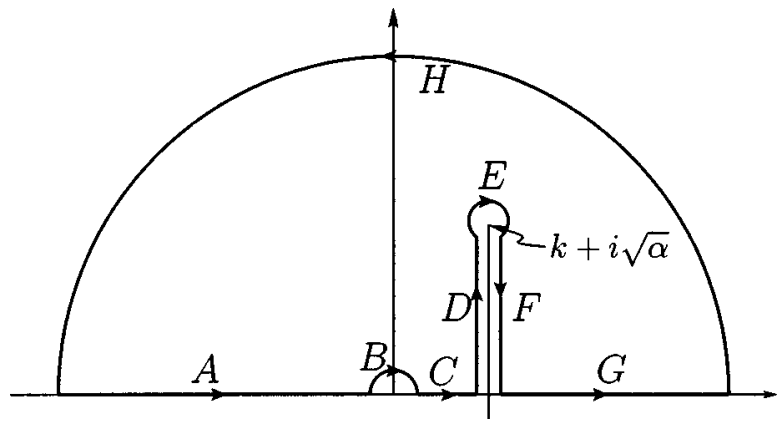

FIG. 4. Contour used in the evaluation of the integral (A7).

$$
\text { a. } \alpha \leqslant 0
$$

For this case, $G(k, \alpha, x)$ can be recast as

$$
\begin{aligned}
G(k, \alpha, x)= & \int_{-\infty}^{\infty} d q \frac{e^{i q x}}{q} \ln \left|\frac{q+(k-\sqrt{-\alpha})}{q-(k-\sqrt{-\alpha})}\right| \\
& +\int_{-\infty}^{\infty} d q \frac{e^{i q x}}{q} \ln \left|\frac{q+(k+\sqrt{-\alpha})}{q-(k+\sqrt{-\alpha})}\right|=S(k-\sqrt{-\alpha}, x) \\
& +S(k+\sqrt{-\alpha}, x) .
\end{aligned}
$$

Yafet $^{20}$ has evaluated an integral of the type above; the result in the present case is expressed in terms of the $S$ function defined in (A1).

\section{b. $\boldsymbol{\alpha}>\mathbf{0}$}

When $\alpha$ is positive, the numerator and denominator of the logarithmic argument has zeros in both the upper and lower half planes of the complex $q$ space. This entails a careful treatment of the branch cuts of the ln function. An integral of the type

$$
I(k, \alpha, x)=\int_{-\infty}^{\infty} d q \frac{e^{i q x}}{q} \ln \left|(q-k)^{2}+\alpha\right|
$$

can be evaluated by noting that

$$
\int_{c} d q \frac{e^{i q x}}{q}[\ln (q-k+i \sqrt{\alpha})+\ln (q-k-i \sqrt{\alpha})]=0,
$$

where $\mathrm{C}=A+B+C+D+E+F+G+H$ is the contour shown in Fig. 4. This relation follows from the fact that for the branch cut for $\ln (q-k-i \sqrt{\alpha})$ is chosen as shown in Fig. 4, the integrand is analytic in the upper half plane avoiding the cut. The branch cut of $\ln (q-k+i \sqrt{\alpha})$ can be chosen such that the contour $\mathrm{C}$ does not cross it. From the relation (A8), it follows that ( $P$ denotes principal value)

$$
\begin{aligned}
I(k, \alpha, x)= & -2 \pi i \oint_{-\infty}^{k} d q \frac{e^{i q x}}{q}-\pi i \oint_{-\infty}^{\infty} d q \frac{e^{i q x}}{q}+\pi i\left(\ln \left|k^{2}+\alpha^{2}\right|\right. \\
& +3 \pi i)+2 \pi \int_{0}^{\sqrt{\alpha}} d \xi \frac{e^{i(k+i \xi) x}}{k+i \xi},
\end{aligned}
$$

where the first two terms are contributions from $A, C$, and $G$ (Fig. 4), the third term is from $B$, and the last from $D$ and $F$. Contributions from $E$ and $H$ vanish. Form a similar expression for $I(-k, \alpha, x)$ [the same as $I(k, \alpha, x)$ with $k$ set to $-k$, except the third term has $\pi i$ instead of $3 \pi i]$, it follows that

$$
G(k, \alpha, x)=2 S(k, x)+R(k, \sqrt{\alpha}, x),
$$

where $S$ and $R$ are functions defined in (A1) and (A2).

\section{c. Asymptotic formulas}

Relations (A6) and (A10) complete the evaluation of the function $G$. It is also useful to record the asymptotic behavior of the $G$ function as $x \rightarrow \infty$. These can be easily inferred from the relations (A3) and (A4).

$$
\begin{aligned}
& G^{a}(k, \alpha, x) \\
& \quad= \begin{cases}2 \pi\left(\frac{\cos (k-\sqrt{|\alpha|}) x}{(k-\sqrt{|\alpha|}) x}+\frac{\cos (k+\sqrt{|\alpha|}) x}{(k+\sqrt{|\alpha|}) x}\right), & \alpha \leqslant 0 \\
0, & \alpha>0,\end{cases}
\end{aligned}
$$

where only terms of $O(1 / x)$ are retained. When $\alpha$ $>0, G(k, \alpha, x) \rightarrow 0$ exponentially as $x \rightarrow \infty$.

\section{The function $H_{m}(x)$}

This function is defined as

$$
\begin{aligned}
H_{m}(x)= & \frac{1}{2} \sum_{n=-N_{f}}^{N_{f}}\left(G\left(k_{f n},(n-m)^{2}-\epsilon_{f}, x\right)\right. \\
& \left.+G\left(k_{f n},(n+m)^{2}-\epsilon_{f}, x\right)\right),
\end{aligned}
$$

where the function $G$ is defined in (A6) and (A10). Asymptotic formulas for $H_{m}$ can be obtained from (A11). It is noted that when $m>N_{f}+\sqrt{\epsilon_{f}}, H_{m}(x)$ tends to zero exponentially as $x \rightarrow \infty$ as is inferred from (A11).
*Electronic address: shenoy@mrc.iisc.ernet.in

${ }^{1}$ S. Ijima, Nature (London) 354, 56 (1991).

${ }^{2}$ R. Saito, G. Dresselhaus, and M. Dresselhaus, Physical Properties of Carbon Nanotubes (World Scientific, Singapore, 1998).

${ }^{3}$ R. Saito, M. Fujita, G. Dresselhaus, and M. S. Dresselhaus, Appl. Phys. Lett. 60, 2204 (1992).

${ }^{4}$ J. W. Mintmire, B. I. Dunlap, and C. T. White, Phys. Rev. Lett.
68, 631 (1992).

${ }^{5}$ N. Hamada, S. I. Sawada, and A. Oshiyama, Phys. Rev. Lett. 68, 1579 (1992).

${ }^{6}$ T. W. Odom, J.-L. Huang, and C. M. Lieber, J. Phys.: Condens. Matter 14, R145 (2002).

${ }^{7}$ S. J. Tans, M. H. Devoret, H. Dai, A. Thess, R. E. Smalley, L. J. Geerlings, and C. Dekker, Nature (London) 386, 474 (1997). 
${ }^{8}$ M. Bockrath, D. H. Cobden, P. L. McEuen, N. G. Chora, A. Zetti, A. Thess, and R. E. Smalley, Science 275, 1922 (1997).

${ }^{9}$ M. Bockrath, D. H. Cobden, J. Lu, A. G. Rinzler, R. E. Smalley, L. Balents, and P. L. McEuen, Nature (London) 397, 598 (1999).

${ }^{10}$ T. Dürkop, B. M. Kim, and M. S. Fuhrer, J. Phys.: Condens. Matter 16, R555 (2004).

${ }^{11}$ P. Avouris, J. Appenzeller, R. Martel, and S. J. Wind, Proc. IEEE 91, 1772 (2003).

${ }^{12}$ T. W. Odom, J.-L. Huang, and C. M. Lieber, Science 290, 1549 (2000).

${ }^{13}$ D. P. Clougherty, Phys. Rev. Lett. 90, 035507 (2003).

${ }^{14}$ F. Wei, J.-L. Zhu, and H.-M. Chen, Phys. Rev. B 67, 125410 (2003).
${ }^{15}$ M. A. Ruderman and C. Kittel, Phys. Rev. 96, 99 (1954).

${ }^{16}$ T. Kasuya, Prog. Theor. Phys. 16, 45 (1956).

${ }^{17}$ K. Yoshida, Phys. Rev. 106, 893 (1957).

${ }^{18}$ J. H. Van Vleck, Rev. Mod. Phys. 34, 681 (1962).

${ }^{19}$ C. Kittel, in Solid State Physics, edited by F. Seitz, D. Turnbull, and H. Ehrenreich (Academic, New York, 1968), Vol. 22, pp. $1-26$.

${ }^{20}$ Y. Yafet, Phys. Rev. B 36, 3948 (1987).

${ }^{21} \mathrm{~K}$. Yoshida, Theory of Magnetism (Springer-Verlag, Berlin, 1998).

${ }^{22}$ J. Kondo, in Solid State Physics, edited by F. Seitz, D. Turnbull, and H. Ehrenreich (Academic, New York, 1969), Vol. 23, pp. 183-281. 